\title{
Methodological Aspects of Controlling Multilevel Man-Machine Organization Systems
}

\author{
Dimitri GOLENKO-GINZBURG ${ }^{1}$, Avner BEN-YAIR ${ }^{2}$, Nitzan SWID ${ }^{3}$
}

${ }^{1}$ Department of Industrial Engineering and Management (Emeritus), Ben-Gurion University of the Negev, Beer-Sheva, Israel \& Department of Industrial Engineering and Management,

Ariel University of Samaria, Ariel, Israel

${ }^{2}$ Center for Reliability and Risk Management, SCE - Shamoon College of Engineering, BeerSheva, Israel

${ }^{3}$ Department of Industrial Engineering and Management, Ariel University of Samaria, Ariel, Israel \& Department of Industrial Engineering and Management, Bar-Ilan University, Ramat-Gan, Israel

11dimitri@ariel.ac.il, ${ }^{2}$ avner2740@gmail.com, ${ }^{3}$ nitzan@effectivy.net

Hierarchical on-line control models for multilevel man-machine organization systems (production and project management systems) are outlined. The models are based on the conception of emergency situations and risk averse on-line control. By using the idea that hierarchical levels can interact only in special situations, the so-called emergency points, one can decompose general and complex multi-level problems of optimal control into sequences of one-level control problems. A hierarchical on-line control model under chance constraint is presented. The model comprises a chance constraint at the upper level and enables at the lower level optimizing both the units' starting time and the resources to be hired. The objective is the average total expenses within the planning horizon while the chance constraint is the minimal permissible probability of meeting the due date on time.

Keywords: Man-Machine Production System, On-Line Production Control, On-Line Project Management, Risk Averse Control Model, Chance Constraint Control Model, Control Via Coordination Principles, Control Via Emergency Situations

1

Introduction

The control models to be considered in this paper are intended both for man-machine production systems and project management systems for which the progress of the systems' advancement towards the goal cannot be inspected and measured continuously, but only at preset inspection (control) points. For all organization units (OU) at the lower system's level (e.g., production units comprising a group of machines, network projects comprising activities, etc.) on-line control has to determine both inspection points and control actions to be implemented at those points to alter the progress of the OU in the desired direction. On-line control is usually carried out to minimize the number of inspection points needed to meet the target, since inspecting the units' output is usually a costly operation. In addition, in certain cases, on-line control for a OU under random disturbances has to be carried out subject to a chance constraint

\section{$[4,6]$.}

The generalized on-line production control model has to be formulated as follows $[4,6]$ : determine both optimal control points $t_{g}$ to inspect the OU and optimal control actions $C A\left(t_{g}, r_{g}\right)$ to be implemented at those control (inspection) points $\left(r_{g}\right.$ being the index of the control action), in order to minimize the number $W$ of inspection points

$$
\left\{\begin{array}{l}
\operatorname{Min} \\
t_{g}, r_{g}
\end{array}\right\}
$$

subject to

$\operatorname{Pr}\left\{t_{g}, r_{g}\right\} \geq p^{*}, t_{0}=0, t_{W}=D,{ }^{t_{g+1}-t_{g} \geq \Delta}$. Here $D$ is the due date and $\operatorname{Pr}\left\{t_{g}, r_{g}\right\}$ is the restricted from below probability of meeting the deadline on time, when introducing

$$
C A\left(t_{g}, r_{g}\right) \text {. }
$$

Note that if implementing a control action 
$C A\left(t_{g}, r_{g}\right)$ results in determining the unit's speed $v_{t_{g}}$ to proceed with until the next inspection point $t_{g+1}$ and if several alternative speeds can be chosen, then the optimal control action enables adopting the minimal speed while honoring chance constraint (2) [4-6].

\section{On-line Production Control Models}

It can be well-recognized [1-6] that control model (1-5) is in fact a stochastic optimization problem with a non-linear chance constraint and a random number of optimized variables. Such a problem is too difficult to solve in the general case. Thus, heuristic control algorithms have been developed [4-6] to determine the next inspection point $t_{g+1}$. Three different classes of algorithms are considered for organization units at the lower level:

I. Using sequential statistical analysis to maximize the time span between two adjacent inspection points $\Delta t_{g}=t_{g+1}-t_{g}$.

II. Using the methodology of a risk-averse decision-maker.

III. Using the methodology of the chance constraint principle.

Algorithm I [6] solves the on-line control problem as follows: to maximize the objective $\left(t_{g+1}-t_{g}\right)$ subject to (3-5) and

$$
\operatorname{Pr}\left\{V_{t} \geq V_{t}^{*}\left(t_{g}\right)\right\} \geq p^{*}, \quad \forall t: t_{g} \leq t \leq t_{g+1} \text {. }
$$

This problem can be solved by determining the maximal value $T^{*}$ satisfying

$$
T^{*}=\underset{t_{g}<t \leq D}{\operatorname{Max}}\left\{t: \psi\left(q_{t}\right) \geq p^{*}\right\} \text {. }
$$

Here

$$
\begin{gathered}
\psi(x)=\frac{1}{\sqrt{2 \pi}} \int_{x}^{\infty} e^{-\frac{u^{2}}{2}} d u \quad q_{t}=\frac{\bar{H}_{t}}{S^{2}\left(H_{t}\right)}, \\
H_{t}=V_{t}-V_{t}^{*}\left(t_{g}\right)
\end{gathered}
$$

while $\bar{H}_{t}$ and $S^{2}\left(H_{t}\right)$ designate the average and variance of random value $H_{t}$, correspondingly. In practice, $T^{*}$ can be calculated by means of simulation with a constant step of length $\Delta$. The procedure of increasing $t$ step-by-step is followed until (7) ceases to hold. The thus determined value $T^{*}$ satisfies $t_{g}+T^{*}=t_{g+1}$. Algorithm $\mathrm{I}$ is outlined in depth in [4-6] (case of a single, fixed speed and case of several alternative speeds).

Algorithm II is based on the concept of riskaverse decision-making [4,6]. Given a routine inspection point $t_{g}$, the unit's output observed at that moment $V_{t_{g}}$ and the control action $C A\left(t_{g}, r_{g}\right)$ to be implemented at moment $t_{g}$ up to the next inspection point, the problem is to determine that next point $t_{g+1}$. As for Algorithm I, the objective is to maximize the time span $\left(t_{g+1}-t_{g}\right)$. Value $t_{g+1}$ is determined so that even if the unit's productivity (speed) is most unfavorable in interval $\left[t_{g}, t_{g+1}\right]$, i.e., with the minimal rate $v^{\prime}\left(t_{g}, r_{g}\right)$, then introducing the most effective control action $C A\left(t_{g+1}, r\right)$ at moment $t_{g+1}$ enables the unit to meet its target on time, subject to the chance constraints. Here $r$ is the index of the most effective control action, e.g., $r$ is the index of the highest possible speed to be introduced. Value $r$ is determined via "risk-averse" heuristics $V_{t_{g}}+v^{\prime}\left(t_{g}, r_{g}\right)\left(t_{g+1}-t_{g}\right)+\bar{v}\left(t_{g+1}, r\right)\left(D-t_{g+1}\right)=V^{*}$ Note that the minimal rate $v^{\prime}\left(t_{g}, r_{g}\right)$ can be substituted for a $p$-quantile of the random speed $v\left(t_{g}, r_{g}\right)$ when the confidence level $p$ is close to zero.

\section{The Chance Constraint Principle}

Both on-line control algorithms are implemented in real time. However, in order to check the validity of any of them, the algorithms' functioning can be simulated. The comparative efficiency of Algorithms I and II has been tested on various examples of medium-size OUs $[4,6]$. A general conclusion 
can be drawn that applying the second algorithm rather than the first results both in essentially smaller computational time and in cheaper unit's productivity. Both methods honor the chance constraint $p^{*}$ and can be implemented for various production control models.

However, both models I and II do not support solving cost-optimization problems. This shortcoming called for the creation of the online control model III which is a costoptimization model and is based on the socalled chance constraint principle [4-6].

Given the average processing costs per time unit for each activity to be operated under each speed, together with the average cost of performing a single inspection at the chosen control point, the problem at a routine inspection point $t_{g}$ is to determine the proper speed $v^{(k)}$ and the next inspection point $t_{g+1}$ , in order to minimize the total processing costs within the planning horizon, subject to a chance constraint. At each inspection point, decision-making centers around the assumption that there is no more than one additional inspection point before the due date. Following that assumption, two speeds $v^{\left(k_{1}\right)}$ and $v^{\left(k_{2}\right)}$ have to be chosen at a routine inspection point $t_{g}$ :

1. Speed $v^{\left(k_{1}\right)}$ which has to be actually introduced at point $t_{g}$ up to the next inspection point $t_{g+1}$;

2. Speed $v^{\left(k_{2}\right)}$ which is forecast to be implemented at inspection point $t_{g+1}$ up to the due date $D$.

The couple $\left(v^{\left(k_{1}\right)}, v^{\left(k_{2}\right)}\right)$ providing the minimal total cost expenses, has to be accepted.
The model is particularly efficient when the unit's output can be measured as a partial accomplishment of the entire planned program.

\section{The Model Based On Emergency Situa- tions}

Mesarovich et al [7] presented a virtual multilevel analytical production control model with hierarchical levels coordinated by means of the interaction balance principle. Each level comprises a variety of complicated optimization models with appropriate linkage. Problems of coordinating optimal planning models in the developed multilevel production system are based on a hierarchical "tree" of local models and corresponding optimization problems. Problems of coordination between models for two contiguous hierarchical levels are solved by implementing the Mesarovich interaction balance principle. The model considers both a sub-model of querying the system elements and determining local (internal) control actions, as well as a sub-model of parametrical (external) control at each hierarchical level. The integrated model incorporates both models of multicriterial optimization for elements of a single hierarchical level, and coordination models for contiguous levels of the hierarchy.

Such a virtual three-level analytical production control model with hierarchical levels coordinated by means of Mesarovich interaction balance principle is very complicated, cannot be reduced to control algorithms and, thus, is unfit to be used in practice. To simplify the control model, we have substituted the interaction balance principle by another one, namely, the conception of emergency situations which has been created within the last two decades. 

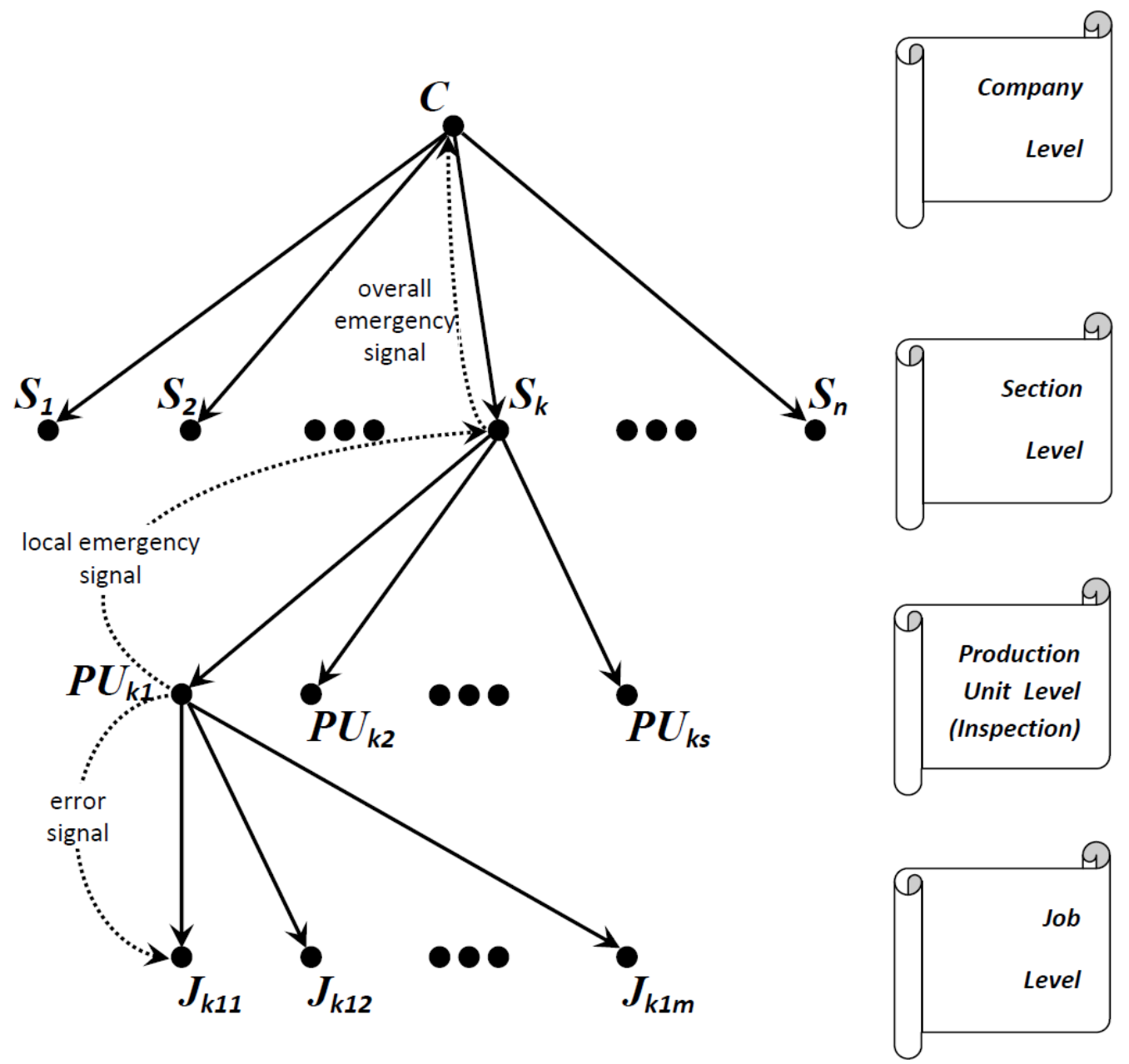

Fig. 1. Hierarchical production control model (error and emergency signals)

The regarded approach to the interaction submodels in hierarchical system, based on the conception of emergency, is as follows. By using the idea that hierarchical levels can interact only in special situations, the so-called emergency points, one can decompose general and complex multi-level problems of optimal production control into sequences of one-level problems.

This approach is demonstrated on Figure 1, where a four-level production system is presented.

Inspection is carried out on the production unit level. If it is anticipated that a certain unit cannot meet its local target on time, an error signal is generated participating in the so-called internal optimal control. The latter is carried out by reallocating and reassigning the remaining unit's target as well as the unit's resources, among the subordinated jobs. If such an optimal control action ena- bles the unit to meet its target on time, the single-level on-line control model starts functioning, i.e., the next inspection point as well as the unit's speed to proceed with are determined. If the internal control does not succeed in enhancing the unit's speed a local emergency signal is declared and external control has to be undertaken at the section level, namely, at the section to which the unit under emergency is subordinated. At the section level the optimal reallocating and reassigning procedure (this time among all the units subordinated to that section) is carried out. If this control action fails, an overall emergency is declared, and optimal reallocation and reassignment at the company level is undertaken. Thus, the general idea of an emergency model is first to undertake internal control, and if the latter fails, to apply the higher hierarchical level, until we either succeed in optimizing the system's parameters or 
have to change the system's target. The outlined above idea has been successfully implemented in several multilevel on-line control models $[4,6]$.

\section{Hierarchical On-Line Production Con- trol Model with Risk-Averse Decision- Making}

In the present section, we present hierarchical on-line control models related to actual production systems. Those models are not based on optimal problems' solutions and, thus, are not aimed at optimal probability control. However, those models incorporate advanced heuristic approaches and cover all practical requirements applicable to real industrial enterprises.

Assume a company (upper hierarchical level) considered to be composed of several sections on the second level. Each section includes, in turn, several production units on the first (lower) level. Each unit:

- is required to produce a given target amount by a given due date common to all units;

- has several possible speeds, which are subject to random disturbances;

- utilizes resources (workers, devices, machine tools, etc.) in the course of the production process.

For some sections resources can be reallocated among the subordinated units in the course of manufacturing, in order to help the slower unit to meet the deadline. Certain sections include units with transferrable target amounts where each unit, when necessary, may produce target amounts of other units entering the same section. Note that both reallocating resources and reassigning target amounts among the units is the sole prerogative of the section management.

Two types of objective functions are considered when optimizing multilevel manmachine production systems:

a) the objective is to maximize the expected net profit for each production unit (section, company) on each level (type A) [46];

b) the objective is to maximize the probability of achieving the target amount on the due date, without taking into account cost parameters (type B). The latter can be modified by adding a second conflicting objective to minimize the number of inspection (control) points [4-6]; yet, out of the two the first objective is dominant.

Type B objective is implemented when controlling certain assembly systems, "just-intime" systems, defense related industries, designing and building unique installations, etc. In all these cases the penalty costs for the shortage at the end of the planning horizon exceed essentially both the manufacturing expenses and the inspection costs. Type $\mathrm{A}$ is usually used in all other cases.

At the unit level, the management has to make timely observations and determine control points (inspection points) to ensure that the production output is on target. At every inspection point, given the target amount, the due date and the actual amount already produced, the decision-maker has to determine both the proper speed and the next inspection point. We have outlined a heuristic algorithm [6] comprising two conflicting objectives: maximizing the probability of completing production on the due date and minimizing the number of inspection points. The algorithm suggests the choice at each inspection point of the minimal speed which on average will ensure completion of the production on time. The next inspection point is determined by assuming that if, in the worst case, the unit advances by the minimal rate (the lower boundary value of the speed chosen) until the next inspection point, then, by applying the maximal speed from that point on, there will still be enough time to meet the deadline. Thus, the on-line algorithm prevents unnecessarily high speeds, and control actions are carried out as rarely as possible but without missing the moment after which the tendency to deviate may cause irreversible delay of the completion time.

At the unit level, each unit works independently according to this algorithm. If a critical situation occurs, i.e., if at any inspection point it becomes clear that for any of the units, even at the maximal processing speed, the target will not be met on the due date, the 
section reschedules the remaining overall target amount among the units in order to help the failing one to meet the deadline. An optimization problem has to be solved at the section level where the optimizing variables are the parts of the remaining target amounts transferred from certain units to others. The objective function is maximizing the probability of completing the overall target amount on the due date. New target amounts are determined for the units, which then proceed to work independently either until the next emergency moment or until reaching the due date.

It is assumed that, for all units, the control time, the speed reset and the target amount rescheduling time are negligible, and that the set-up costs are fixed independently of the speed and of the unit. Further, we assume that the speed is a random variable; namely, it varies at the routine inspection point and remains constant until the next inspection point.

It can be well-recognized that such decisionmaking is adequate to most of the semiautomated production systems. If, e.g., a building project is carried out by means of several teams, the project manager may reassign, if necessary, some activities or subprojects from a slower team to faster ones, so that the entire project will be accomplished on time.

An emergency is called at the company level at moment $t$ if one of the sections cannot accomplish its target on time, i.e., there is no feasible solution to the section optimization problem. Even if the units help each other, the target will not be met on time. In this case, given the actual amount produced at $t$ by each section, the company will have either to reallocate the total capacity of resources among the sections, or to reassign the company target amount among the sections [4-6].

\section{Multilevel On-Line Control Models Based On Chance-Constraint Principle}

In the previous section we have used the general idea that hierarchical levels can interact only in special situations, the so-called emergency moments. We have decomposed a general and complex multilevel problem of optimal production control into a sequence of one-level problems.

The model outlined above does not implement a chance constraint in the on-line production control model. In our opinion, minimizing the system's expenses to meet the target on time, i.e., at a given due date, is not to be the only goal in the course of the longterm cooperation with various customers. To honor the company's good name, an additional requirement has to be inserted in the model: the production system has to meet its due date on time with a pregiven confidence probability. Thus, a chance constraint has to be implemented in the control model.

The outlined below multilevel control model under a chance constraint [6] is a further development of the model presented in Section 3. A control model for a three-level manmachine production system under random disturbances is considered. The system comprises the factory level, several sections and multiple production units. The factory is faced with manufacturing several different products with planned target amounts at the given due date. Each unit can manufacture all kinds of products by utilizing different types of renewable resources. In order to introduce control actions all production units have to be inspected at control points to observe the output of the products. The problem is to develop control models at each production level, in order to minimize the factory's average total expenses under a chance constraint. The optimal variables are the starting time of the manufacturing process and the capacities of all types of resources to be hired throughout the planning horizon. Two models are developed at the factory level: a search coordinate descent model for optimized variables and a reallocation model to redistribute both the resources and the planned target among the sections. At the section level a hierarchical "tree" of optimal reallocation models to redistribute both the resources and the target amounts among the subordinated elements at the adjacent lower level will be considered. At the unit level control actions boil down to determining inspection points and production 
speeds. All the regarded optimal control models are imbedded in a three-level simulation model which provides representative statistics in order to assess the efficiency of the outlined factory model.

The main difference between the hierarchical model under consideration and the model outlined in Section 5 is as follows:

1. The cost objective at the upper level in the model outlined in Section 5 is to minimize the budget for the resource consumption within the planning horizon. In the present hierarchical model the cost objective at the upper level is to minimize all the expenses connected with the manufacturing process, e.g., the processing costs (for different speeds), the costs of performing inspection at the manufacturing level, the costs of reallocating resources and target amounts at different levels, the penalty cost to the factory for not accomplishing the total production program at the due date $D$, etc.

2. Minimizing the total factory expenses to manufacture the product's required amounts at the given due date, has not to be the only factory's goal in the course of a long-term cooperation with various customers. In order to honor the company's good name, an additional requirement has to be introduced in the model, namely, to guarantee the products' delivery performance, i.e., the completion of the production program at the due date with a pregiven confidence probability. This means that an additional chance constraint has to be implemented in the model.

3. In the models outlined in Section 5 the optimized values to be determined are restricted by the total resource capacities at the factory's disposal (to be hired and utilized within the planning horizon). However, other parameters to be optimized may be imbedded in the model. Given the due date to complete the production program, the starting time to begin the manufacturing process refers to the optimized variables as well.

The conceptions outlined above are imbedded in the structure of the hierarchical three- level control model. At the upper (factory) level a quasi-optimal, heuristic search model to determine the optimal resource amounts to be hired together with the optimal starting time to realize the manufacturing process, is introduced (call it Model $A_{1}$ ). A cost objective to minimize the average total manufacturing expenses, subject to a chance constraint of meeting the pregiven due date on time, is imbedded in the model.

An optimal model (Model $A_{2}$ ) at the upper level reallocates the resources together with the target amounts among the sections at the second level. Another coordinated and balanced with model $A_{2}$ reallocation model at the second level (Model $B_{1}$ ) redistributes both the resources and the products' target amounts among the production units at the lower level. A heuristic model to determine both routine control (inspection) points and production speeds to proceed with until the next control point (Model $C_{1}$ ), is imbedded in the model at the manufacturing level.

All models A, B, C are outlined in depth in [6].

\section{Methodological Aspects for the Case of a Hierarchical Stochastic Network Project}

The approach of controlling multilevel systems via emergency situations for the case of stochastic project management is outlined in the present section.

The presented hierarchical model combines together two resource reallocation models at the upper level, the on-line control model at the medium level and a resource supportability model at the lower level.

Several activity-on-arc network projects (graphs) with independent activities of random durations are considered. Each activity duration follows a beta probability density function while the cost-duration function is based on the assumption that each activity duration is close to be inversely proportional to the budget assigned to that activity.

A hierarchical control model is suggested [4] which at any control point determines:

- optimal budget values assigned from the company to each project, 
- optimal budget reallocation among the project's activities,

- optimal control points to inspect each project,

in order to

- minimize the total number of control points for all projects, and

- maximize the probability of meeting the deadline of the slowest project.

The model is based on a stochastic optimization problem with two conflicting objectives and a variable number of constraints. The problem cannot be solved in the general case and allows only heuristic solutions. The general control model is modified to the hierarchical on-line control model, which comprises three optimization problems. Problem I, at the company level, enables optimal budget reassignment among the projects. The problem's solution, i.e., the budget assigned to each project, serves as the initial data for Problem II (at the project level), where budget is reallocated among the project's activities to maximize the probability of meeting the project's deadline. The solution of Problem II serves, in turn, as the initial data for Problem III, which carries out on-line control, i.e., determines optimal control points to inspect the progress of the project. This is done by determining the planned trajectories that must be repeatedly corrected in the course of the project's realization.

If, at any control point, it turns out that a project deviates from the planned trajectory, an error signal is generated, and decisionmaking is based on solving Problem II to reassign the remaining budget among the remaining project's activities to maximize the probability to meet the deadline. If the problem's solution enables the project's deadline to be met, subject to the chance constraint, a corrected planned trajectory is determined, and Problem III is resolved to determine the next control point. Otherwise an emergency signal is generated, and decision-making is carried out at the company level. Problem I is resolved under emergency conditions to reassign the remaining budget among the nonaccomplished projects. Thus, in the course of controlling a group of projects, the latter are first optimized on line from "top-to-bottom". In the case of an emergency, the generated "bottom-top" signals are converted into control actions to enable the projects' due dates to be met on time.

Let us introduce the following terms:

\section{The company level}

$C$ - the total company budget assigned at $t=0$ for all project's realization;

$n$ - number of projects;

$G_{k}(N, A)$ - the $k$-th stochastic network project (graph) of PERT-COST type, $1 \leq k \leq n$; $C_{k} \quad$ - budget assigned to the $k$-th project at moment $t=0$;

$C_{k t} \quad$ - budget assigned to the $k$-th project at moment $t>0$;

$C_{k}(t)$ - the remaining project's budget at moment $t \geq 0$ (observed via inspection), $C_{k}(0)=C_{k}$.

$D_{k} \quad$ - the due date for the $k$-th project;

$P_{k}^{*} \quad$ - the pregiven minimal possible probability for the $k$-th project to meet it's deadline on time;

$G_{k t} \quad$ - the remaining part of graph $G_{k}(N, A)$ at moment $t \geq 0 ; G_{k 0}=G_{k}$;

$T_{k t}\left\{C_{k}(t)\right\}$ - the random duration of $G_{k t}$ on condition that at $t \geq 0$ the remaining budget is $C_{k}(t)$;

$P_{k t}=P\left\{C_{k}(t)\right\}$ - the probability of meeting the project's $G_{k t}$ deadline on time on condition that at moment $t \geq 0$ the remaining budget is $\quad C_{k}(t), \quad$ i.e., $P_{k t}=P\left\{t+T_{k t}\left\{C_{k}(t)\right\} \leq D_{k}\right\}$;

$C(t)$ - the remaining company's budget at moment $t>0$.

\section{The project level}

For simplicity we will omit index $k$ : $(i, j) \in G(N, A)$ - activity entering the project; 
$t_{i j} \quad$ - random duration of $(i, j)$;

$c(i, j)$ - budget assigned to activity $(i, j)$;

$c(i, j)_{\min }$ - the minimal budget with which ac-

tivity $(i, j)$ can be operated (pregiven);

$c(i, j)_{\max }$ - the maximal budget to operate $(i, j)$ (pregiven);

$t_{i j}\{c(i, j)\}$ - the random duration of activity $(i, j)$ on condition that budget $c_{i j}$ is assigned to $(i, j), c(i, j)_{\min } \leq c(i, j) \leq c(i, j)_{\max }$;

$S_{i j} \quad$ - the moment $(i, j)$ actually starts;

$T_{i j}^{r} \quad$ - the resource delivery moment for activity $(i, j)$ (a random value, which is determined in the course of the project's realization);

$F_{i j}=S_{i j}+t_{i j}$ - the moment activity $(i, j)$ is accomplished;

$V$ - target amount for the project of PERTCOST type; let $V=C$, where $C$ is the budget assigned to the project;

$V^{t} \quad$ - the actual realized part of target amount $V$ at moment $t>0$; for PERTCOST projects $V^{t}=C^{t}$, where $C^{t}$ is the budget actually realized at moment $t$;

$C(t)=C-C^{t}$ - the remaining (non-realized) budget at moment $t>0$ (observed via inspection);

$T_{r}(x, t, D)$ - the control trajectory for the project determined at moment $t \geq 0$; this is a straight line connecting points $\left(t, C^{t}\right)$ and $(D, 0)$;

$N$ - number of control (inspection) points in the course of controlling the project; $P^{*} \quad$ - the pregiven minimal confidence probability of meeting the deadline on time;

$t_{g} \quad$ - the $g$-th control point, $g=0,1, \ldots, N ; t_{0}=0, t_{N}=D ;$

$\Delta \quad$ - the minimal time span between two consecutive control points (pregiven for each project);

\section{Optimization Problem I at the Company Level}

At moment $t=0$ the problem is as follows: determine values $C_{k}$ assigned for each project $G_{k}(N, A), 1 \leq k \leq n$, to maximize

$$
J=\underset{C_{k}}{\operatorname{Max}} \operatorname{Min}_{k}\left\{P\left\{C_{k}\right\}\right\}
$$

subject to

$$
\sum_{k=1}^{n} C_{k}=C
$$

and

$$
P\left\{C_{k}\right\} \geq P_{k}^{*}
$$

Problem (10-12) is a very complicated problem which does not obtain a precise solution. Its heuristic solution is outlined in [4].

The corresponding dual problem for the case of one project centers on determining the minimal budget $C$ with pregiven due date $D$ and minimal confidence probability $P^{*}$. One has to determine

Min C

subject to

$$
\operatorname{Pr}\{C\} \geq P^{*}
$$

At moment $t>0$ problem (10-12) is as follows: determine the newly corrected values $C_{k t}^{*}$ to maximize

$$
\underset{\left\{C_{k t}\right\}}{\operatorname{Max}} \operatorname{Min}_{k}\left\{P\left\{C_{k t}\right\}\right\}
$$

subject to

$$
\begin{aligned}
& \sum_{k=1}^{n} C_{k t}^{*}=C(t) \\
& P\left\{C_{k t}^{*}\right\} \geq P_{k}^{*}, \quad 1 \leq k \leq n
\end{aligned}
$$

where $C(t)$ is the remaining company budget which has to be redistributed among the projects.

\section{Optimization Problem II at the Project Level}

For Problem II the input parameters are either $C_{k}$ or $C_{k t}$, which, for the sake of simplicity, are designated by $C$ or $C_{t}$. Thus, index $k$ is further on omitted.

The problem is as follows: to redistribute $C$ among the project's activities in order to ob- 
tain the maximal $P\{C\}$, i.e., to determine values $c(i, j)$

$$
\begin{aligned}
& \underset{c(i, j)}{\operatorname{Max}}[P\{C\}]=\operatorname{Max}_{c(i, j)}[\operatorname{Pr}\{T\{C\} \leq D\}] \\
& \text { subject to } \\
& \quad P\{C\} \geq P^{*} \\
& \quad \sum_{(i, j)} c(i, j)=C \\
& \quad(i, j) \in G(N, A)
\end{aligned}
$$

and

$$
c(i, j)_{\min } \leq c(i, j) \leq c(i, j)_{\max }
$$

Problem (18-22) is solved by using a combination of heuristic procedures and simulation modeling. Note that problem (18-22) is in fact a simplified version of problem (13-14). If $t>0$, the problem can be modified to a more complicated version

$$
\underset{c(i, j)}{\operatorname{Max}}[\operatorname{Pr}\{t+T\{C(t)\} \leq D\}]
$$

subject to (19-22).

After determining values $\{c(i, j)\}$ control points $t_{g}$ have to be determined.

\section{On-line Control Problem III}

The problem [4] is to determine control points $t_{g}, g=0,1, \ldots, N$, which deliver the minimum of the number of those points

$$
\operatorname{Min}_{\left\{t_{g}\right\}} N
$$

subject to

$$
\begin{aligned}
& \operatorname{Pr}\left\{C_{D}=0\right\} \geq P^{*} \\
& t_{g+1}-t_{g} \geq \Delta \\
& t_{1}=0, t_{N}=D
\end{aligned}
$$

Problem (24-27) is a very complicated problem of non-linear stochastic programming. The problem can be solved by substituting it for another one, i.e., to maximize the time span between two consecutive control points. The problem is to determine values $\left\{t_{g}\right\}$ in order to maximize

$$
\operatorname{Max}\left\{t_{g+1}-t_{g}\right\}
$$

subject to

$$
t_{g+1}-t_{g} \geq \Delta
$$

$$
\operatorname{Pr}\left\{C_{t}<T_{r}\left(t, t_{g}, D\right)\right\} \geq P^{*}
$$

In (30) trajectory $T_{r}\left(t, t_{g}, D\right)$ is a straight line connecting two points $\left(t_{g}, C^{t_{g}}\right)$ and $(D, 0)$. The trajectory line is as follows:

$$
T_{r}\left(t, t_{g}, D\right)=\frac{t \cdot C^{t_{g}}}{D-t_{g}}+\frac{D \cdot C^{t_{g}}}{D-t_{g}}=C^{t_{g}} \frac{t+D}{D-t_{g}}
$$

Problem (28-30) has been solved in [4-6] by a combination of statistical sequential analysis and simulation.

If $C^{t_{g+1}} \leq T_{r}\left(t_{g}, t_{g+1}, \Delta\right)$ holds, that means that the project does not deviate from its target and there is no need in any additional control actions. In case $C^{t_{g+1}}>T_{r}\left(t_{g}, t_{g+1}, \Delta\right)$ one has to resolve problem (18-22) for the remaining part of the budget $C\left(t_{g+1}\right)=C-C^{t_{g+1}}$ and the remaining project $G_{t_{g+1}}(N, A)$. The problem results in maximizing the probability of meeting the target on time by rescheduling the budget among the remaining activities. If in the course of solving problem (18-22) we obtain $P\left\{C\left(t_{g+1}\right)\right\} \geq P^{*}$, that means that a new trajectory has to be developed. Thus, a new control point $t_{g+2}$ is obtained, and the project's realization proceeds. If relation $P\left\{C\left(t_{g+1}\right)\right\}<P^{*}$ holds, that means that the project is unable to meet its target on time and needs help from the company.

\section{Conclusions}

The following conclusions can be drawn from the paper:

I. The results obtained include methodological conceptions in order to create a multilevel control model covering all levels of hierarchy - from a single-level element to three-four- level companies - for monitoring complicated organization systems. Such a control model has to comprise a variety of coordinated optimization models under random disturbances.

II. The backbone of the paper are three hierarchical control models based on the con- 
ception of emergency situations and comprising, correspondingly, a risk averse type on-line production control model (Section 5), a chance constraint model (Section 6) and a hierarchical project management control model (Section 7).

III. For the case of a hierarchical project management system all types of optimization models from bottom to top are outlined in depth. This gives an opportunity to overview the control models' functioning.

IV. In the course of controlling a multilevel organization system the latter is first optimized on-line from bottom to top until at one of the upper levels the plan is corrected to ensure that all the subordinated "top-bottom" elements of the system will meet their deadlines. Afterwards, the corrected plan is detailed for those elements, up to the bottom (unit) level. These basic principles can be applied to any hierarchical system, independently of the number of levels. Since the target-amount reassignment problem, although being very efficient, results in more significant alterations in the plan's structure and is essentially more complicated (especially on the upper levels), than the resource reallocation optimization problem, it is recommended, whenever possible, that the latter problem be applied only.

$\mathrm{V}$. Unfortunately, the optimization problems outlined above when solved at various hierarchical levels are not coordinated. For example, when monitoring a multilevel production system, it can be wellrecognized that solving optimization problems at a section level for reallocating resources or reassigning target amounts among subordinated units certainly leads to corrections of specific parameters for these units. However, such corrected characteristics should be "linked" to corresponding parameters at the section level. Alternatively, changes in the latter can require the solution of optimization problems at higher levels, connecting further the solutions of the problems to the goal characteristics of the industrial plant as a whole. The latter can be facilitated by implementing Mesarovich coordination and interaction balance principles [7], which have to be applied to optimization problems at various hierarchical levels.

VI. Thus, in our opinion, the problem of creating a modern improved hierarchical on-line organization control model in order to bring together a higher level of model's optimality with simplicity in structure and in usage, is one of the most urgent problems of future research in industrial engineering. Such a research has to be centered on unification of the interaction balance principle of Mesarovich [7] and the principle of emergency situations $[4,6]$. Both principles have to be combined in a unified hierarchical on-line control model for multiple products and resources. Unfortunately, the problem is as yet far from being solved.

\section{References}

[1] D.I. Golenko, "Analyzing and synthesizing production systems by simulative modeling," in Proc. The $5^{\text {th }}$ International Conference on Collective Phenomena, vol. 410, New-York, U.S.A., 1983, pp. 227-236.

[2] D.I. Golenko, "Concerning the synthesis of optimal planning models in multilevel production systems (Part I)," in Proc. The $5^{\text {th }}$ International Conference on Collective Phenomena, vol. 410, New-York, U.S.A., 1983, pp. 237-241.

[3] D.I. Golenko, "Concerning the synthesis of optimal planning models in multilevel production systems (Part II)," in Proc. The $5^{\text {th }}$ International Conference on Collective Phenomena, vol. 410, New-York, U.S.A., 1983, pp. 242-248.

[4] D. Golenko-Ginzburg, V. Burkov and A. Ben-Yair, Planning and Controlling Multilevel Man-Machine Organization Systems under Random Disturbances, Ariel University Center of Samaria, Ariel: Elinir Digital Print, 2011.

[5] D. Golenko-Ginzburg, Hierarchical Control Models of Man-Machine Production Systems, Vol. 1: Fundamentals, Lorman, 
MS: Science Book Publishing House, 2012.

[6] D. Golenko-Ginzburg, Hierarchical Control Models of Man-Machine Production Systems, Vol. 2: Algorithms and Practical Applications, Lorman, MS: Science Book
Publishing House, 2013.

[7] M.D. Mesarovich, D. Mako and Y. Takahara, Theory of Hierarchical Multilevel Systems, New-York: Academic Press, 1970.

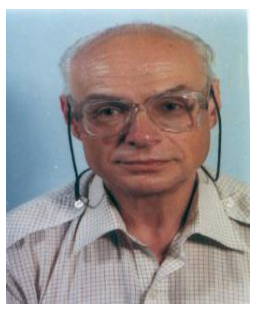

Dimitri GOLENKO-GINZBURG has graduated the Department of Economics at the Moscow Institute of National Economics in 1954. In 1958, he also graduated the Department of Mathematics at the Moscow State University. He holds a PhD diploma in Applied Mathematics from 1962 and has been awarded a Doctor of Technical Sciences diploma by the Chief Degree Awarding Council of the USSR (1966), as well as a Professor diploma in 1968. After immigrating to Israel in 1985, he has been visiting professor in 1986 and full professor in 1988 at the Ben-Gurion University of the Negev in Beer-Sheva (Professor-Emeritus since 2004). Currently he is full Professor within the Department of Industrial Engineering and Management at the Ariel University of Samaria, Ariel. He is also Foreign Member of the Russian Academy of Natural Sciences (2007), Honorary Member of the Russian Project Management Association (SOVNET, 2007). He is author of more than 22 books and over 500 journal articles in the field of project management, organization systems control under random disturbances, and more.

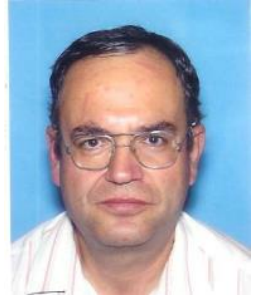

Avner BEN-YAIR has graduated the Department of Health and Safety Engineering and Management of the Ben-Gurion University of the Negev in Beer-Sheva in 2001. He holds a PhD diploma in Industrial Engineering and Management from 2004. Within 2002-2010 has been lecturer and senior lecturer at SCE - Shamoon Academic College of Engineering in Beer-Sheva. He is author of about 50 journal articles in the field of economic aspects of safety, trade-off optimization models for organization systems, production planning, scheduling and control.

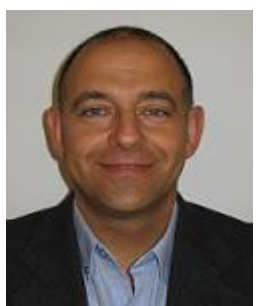

Nitzan SWID is a PhD Candidate at the Department of Industrial Engineering and Management at the Ariel University of Samaria, Ariel, as well as in Department of Industrial Engineering and Management at the Bar-Ilan University in Ramat-Gan, Israel. He is department coordinator of final graduate projects in Ariel; his main research interests include resource reallocation models for deterministic network construction projects. 